\title{
Rapid and Accurate Assembly Method for a New Laue Lens Prototype
}

Wade, Colin; Barriere, Nicolas; Hanlon, Lorraine; Boggs, Steven E.; Brejnholt, Nicolai F.; Massahi, Sonny; Tomsick, John A.; von Ballmoos, Peter

\section{Published in:}

Proceedings of SPIE

Link to article, DOI:

$10.1117 / 12.2187029$

Publication date:

2015

Document Version

Publisher's PDF, also known as Version of record

Link back to DTU Orbit

Citation (APA):

Wade, C., Barriere, N., Hanlon, L., Boggs, S. E., Brejnholt, N. F., Massahi, S., Tomsick, J. A., \& von Ballmoos, P. (2015). Rapid and Accurate Assembly Method for a New Laue Lens Prototype. In Proceedings of SPIE (Vol. 9603). [960309] SPIE - International Society for Optical Engineering. Proceedings of SPIE - The International Society for Optical Engineering https://doi.org/10.1117/12.2187029

\section{General rights}

Copyright and moral rights for the publications made accessible in the public portal are retained by the authors and/or other copyright owners and it is a condition of accessing publications that users recognise and abide by the legal requirements associated with these rights.

- Users may download and print one copy of any publication from the public portal for the purpose of private study or research.

- You may not further distribute the material or use it for any profit-making activity or commercial gain

- You may freely distribute the URL identifying the publication in the public portal 


\title{
Rapid and Accurate Assembly Method for a New Laue Lens Prototype
}

\author{
Colin Wade ${ }^{a, b}$, Nicolas Barrière ${ }^{b, c}$, Lorraine Hanlon $^{a}$, Steven E. Boggs ${ }^{b}$, Nicolai F. Brejnholt ${ }^{d}$, \\ Sonny Massahi ${ }^{e, b}$, John A. Tomsick ${ }^{b}$, Peter von Ballmoos ${ }^{f}$ \\ ${ }^{a}$ School of Physics, University College Dublin, Belfield, Dublin 4, Ireland; \\ ${ }^{b}$ Space Sciences Laboratory, 7 Gauss Way, University of California, Berkeley, CA 94720-7450, \\ USA; \\ ${ }^{c}$ Cosine Research BV, J.H. Oortweg 19, Leiden, 2333 CH, Netherlands; \\ ${ }^{d}$ Lawrence Livermore National Laboratory, 7000 East Ave., Livermore, CA 94550-9234, USA; \\ ${ }^{e}$ National Space Institute, Technical University of Denmark, Elektrovej, 2800, Kgs. Lyngby, \\ Denmark \\ Institut de Recherche en Astrophysique et Planetology, UMR 5277, 9 av. du Colonel Roche, \\ 31028 Toulouse, France
}

\begin{abstract}
The Laue lens is a technology for gamma-ray astrophysics whereby gamma-rays of particular energies can be focused by a suitable arrangement of crystals. The Laue lens assembly station at UC Berkeley was used to build a technological demonstrator addressing the key issues of crystal mounting speed, crystal position and orientation accuracy, and crystal reflectivity. The new prototype is a lens segment containing a total of $485 \times 5$ $\mathrm{mm}^{2}$ crystals - 36 Iron and 12 Aluminium. The segment is composed of 8 partial rings, each of which is aligned to diffract an energy between 95 and $130 \mathrm{keV}$ from a source at $12.5 \mathrm{~m}$ with a focal length of $1.5 \mathrm{~m}$.
\end{abstract}

Keywords: Telescope, Laue lens, Soft Gamma-rays, Crystals, Diffraction, Focusing optics, Technological development

\section{INTRODUCTION}

The study of high energy ( $>50 \mathrm{keV}$ ) emission lines gives insight to the underlying physics of various astrophysical sources. The study of supernovae, in particular, has the potential for a lot of fundamental science through the study of high energy emission lines. ${ }^{44} \mathrm{Ti}$ lines $(78.4,67.9$ and $1157 \mathrm{keV})$ observed in CAS A by COMPTEL ${ }^{1}$ and ${ }^{56} \mathrm{Co}$ lines (847 and $1238 \mathrm{keV}$ ) observed in SN2014J by SPI on INTEGRAL ${ }^{2,3}$ represent the forefront of research in this field of astrophysics. The current generation of space-based telescopes capable of astrophysical line studies is near the limit of the available technology. Compton telescopes (e.g. COMPTEL ${ }^{4}$ ) and coded mask instruments (e.g. SPI on INTEGRAL ${ }^{5}$ ) achieve greater signal by increasing their collecting area but the noise of these instruments grows with the volume of the detector. These factors combine to limit future progress with either kind of instrument since the volumes required for increased sensitivities quickly become impractical for space-borne missions.

To generate larger collecting area without significantly changing the detector size, focusing optics are required. Grazing-incidence optics (e.g. NuSTAR ${ }^{6}$ which can measure energies up to $80 \mathrm{keV}$ ) become less practical at high energies due to the large focal lengths involved. An alternative focusing element is the Laue lens which uses Bragg diffraction to focus photons. By arranging diffracting crystals in a ring and orienting them to diffract a particular energy, a focal spot is created. Adding concentric rings, each either diffracting the same energy (to increase the effective area for that energy) or different energies (to broaden the energy bandpass for the lens), increases the sensitivity of the instrument without altering the volume of the detector.

Further author information: (Send correspondence to C.W. or N.B.)

C.W. E-mail: colin.wade@ucdconnect.ie

N.B. E-mail: nbarriere@cosine.nl

Optics for EUV, X-Ray, and Gamma-Ray Astronomy VII, edited by Stephen L. O'Dell, Giovanni Pareschi, Proc. of SPIE Vol. 9603, 960309 - (C) 2015 SPIE · CCC code: 0277-786X/15/\$18 · doi: 10.1117/12.2187029 
Laue lenses have been under development for several decades. ${ }^{7,8}$ The first diffraction lens with broad energy bandpass was tested onboard a stratospheric balloon in the sixties, with mixed results. ${ }^{9}$ Later projects demonstrated the Laue lens concept as a viable astronomical instrument. One example is the CLAIRE instrument with its observation of the Crab Nebula during a balloon flight in $2001 .{ }^{10}$ Since the concept's inception, work has been ongoing to tackle the two major challenges in constructing a Laue lens: generating a catalogue of usable materials ${ }^{11}$ and developing an accurate assembly method. ${ }^{12}$

A dedicated x-ray beamline at the Space Sciences Laboratory, UC Berkeley has been developed to address the assembly issue. The assembly method consists of two major elements. The first is an x-ray generator that is used to align a given set of crystals and the substrate. The second element is the gluing process used to rapidly and reproducibly mount the crystals on to the substrate. This paper focuses on the most recent developments of this process and the latest prototype, constructed in November 2014.

The Laue lens concept is explained in section 2, the experiment is described in section 3 , the results are presented in section 4 and discussed in section 5 .

\section{LAUE LENS CONCEPT}

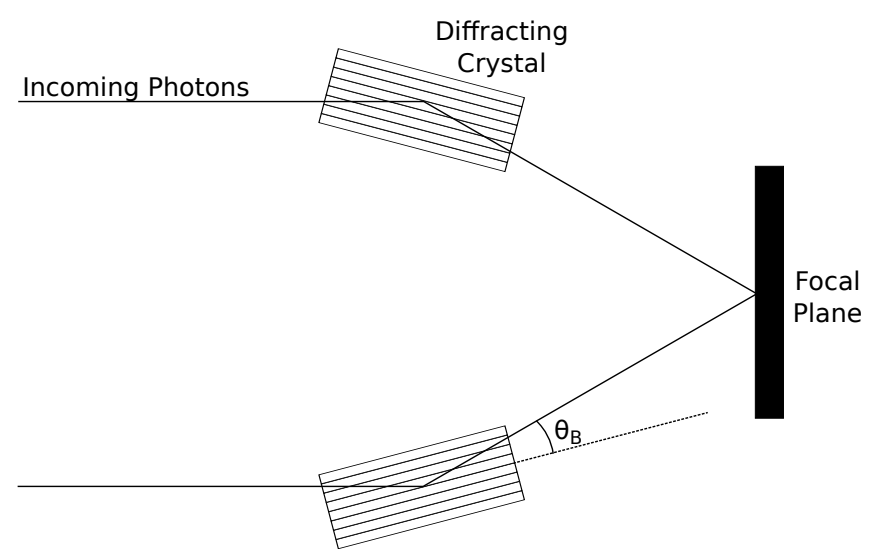

Figure 1. Schematic representation of the principle of the Laue lens. Two crystals diffract photons to a common point.

The principle concept of the Laue lens is to use Bragg diffraction to concentrate photons onto a focal plane. Figure 1 illustrates parallel incoming photons being focused to a single point. Expanding this idea to a ring of crystals, a large collecting area can be generated. Each crystal in the ring will diffract the same energy at the same angle as defined by the Bragg condition:

$$
n \lambda=2 d_{h k l} \sin \theta_{B}
$$

where $n$ is the order of the reflection, $\lambda$ is the wavelength of the photon, $d_{h k l}$ is the inter-planar spacing between the diffracting crystal planes $[\mathrm{hkl}]$ and $\theta_{B}$ is the Bragg angle. To construct a Laue lens with a bandpass, concentric rings of crystals can be oriented to diffract different energies.

Instead of perfect crystals which can only diffract a single energy at a given orientation, mosaic crystals are often used in Laue lens designs. Mosaic crystals can be modelled as being composed of microscopic perfect crystals each slightly misaligned with respect to one another allowing a single macroscopic crystal to simultaneously satisfy the Bragg condition for a range of energies. ${ }^{13,14}$

\section{EXPERIMENT}

\subsection{Crystals}

The materials, orientations and intended central energies for each ring of crystals in the prototype are listed in Table 1. A total of 48 mosaic crystals were glued, 12 aluminium and 36 iron all provided by Mateck. The aluminium crystals are $5 \times 5 \times 4 \mathrm{~mm}^{3}$ while the iron crystals are $5 \times 5 \times 3 \mathrm{~mm}^{3}$. 


\begin{tabular}{ccccc}
\hline Ring & $\begin{array}{c}\text { Radius } \\
\mathrm{mm}\end{array}$ & Material & $\begin{array}{c}\text { Orientation } \\
{[\mathrm{hkl}]}\end{array}$ & $\begin{array}{c}\text { Energy } \\
\text { keV }\end{array}$ \\
\hline 0 & 65.00 & $\mathrm{Al}$ & {$[111]$} & 109.261 \\
1 & 70.15 & $\mathrm{Al}$ & {$[111]$} & 101.250 \\
2 & 75.30 & $\mathrm{Fe}$ & {$[110]$} & 108.694 \\
3 & 80.45 & $\mathrm{Fe}$ & {$[110]$} & 101.749 \\
4 & 85.60 & $\mathrm{Fe}$ & {$[110]$} & 95.640 \\
5 & 90.75 & $\mathrm{Fe}$ & {$[200]$} & 127.598 \\
6 & 95.90 & $\mathrm{Fe}$ & {$[200]$} & 120.764 \\
7 & 101.05 & $\mathrm{Fe}$ & {$[200]$} & 114.627 \\
\hline
\end{tabular}

Table 1. Details of the crystals used to construct the prototype.

\subsection{Substrate}
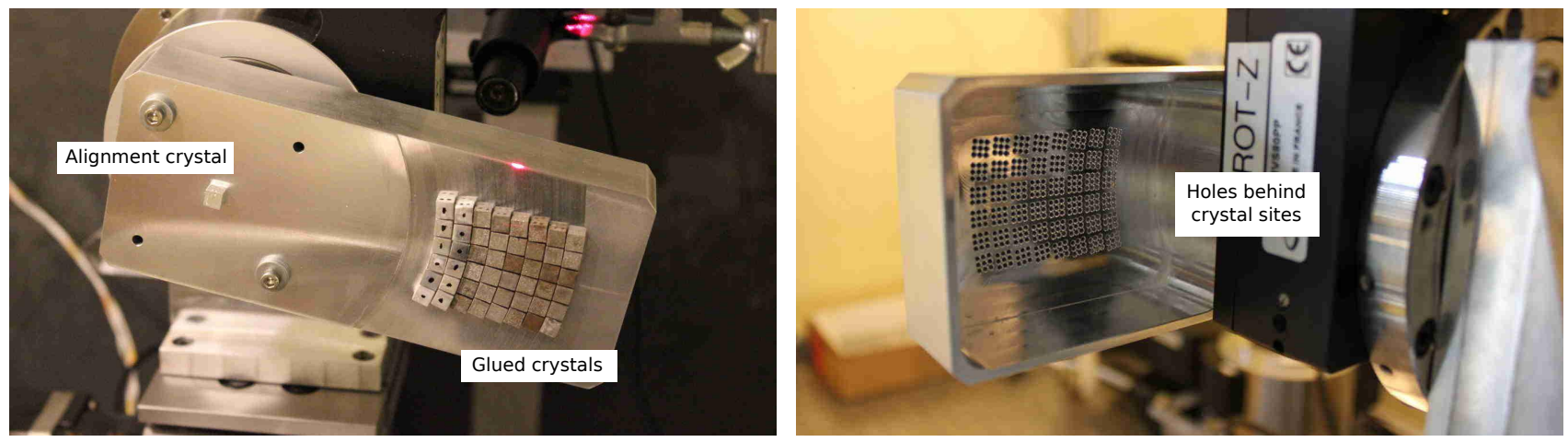

Figure 2. Left: Completed substrate with 48 crystals glued and central alignment crystal. Right: Behind the substrate showing 9 holes for each crystal. These holes allow the UV lamp to illuminate the glue for curing.

Figure 2 shows the completed aluminium substrate. Behind the crystal gluing positions, the aluminium is hollowed out to minimize the absorption of the beam by the substrate. An alignment crystal is used to align the rotational axis of the substrate with the beam axis. When fully aligned, this crystal diffracts the same central energy regardless of the substrate's rotational position. ${ }^{15}$

Crystals on the substrate must be at an angle to the beam to satisfy the Bragg condition. On a flat surface, perpendicular to the beam, this would create a wedge of glue. Upon curing, a wedge can misalign the crystal due to shrinkage. To compensate for this, the substrate was precision machined so that each ring would be at the Bragg angle with respect to the flat of the substrate. However, the surface of the crystal is not necessarily parallel to the relevant crystal plane. The crystals are cut to an accuracy of $\pm 1^{\circ}$. This means that there will still be a wedge of glue and misalignment is still likely to occur while curing due to shrinkage.

The bondline thickness for the glue is $\sim 100 \mu \mathrm{m}$. Illuminating the glue from the side is not a viable option due to the small area available for exposure and the long depth the photons would need to transmit through. Figure 2 shows the holes through the substrate which allow the UV lamp to cure the glue from behind the crystal when it is against the substrate surface.

\subsection{Beamline}

A detailed description of the experimental setup is given in previous proceedings. ${ }^{16}$ Updates to the setup include:

- Improved thermal insulation.

- Replacing manually controlled stages with motorized stages for aligning the substrate. 
- Redesigning the stack of stages controlling the crystal position such that the crystal is at the centre of rotation for each of alignment motors. This ensures the crystal is kept within the beam while being aligned.

- Attaching a distance sensor to measure the distance between crystal and substrate.

- Attaching a mirror behind the substrate and a digital collimator tracking the substrate orientation to check for any misalignment caused by moving the substrate.

- Implementing a UV lamp behind the substrate for curing the glue.
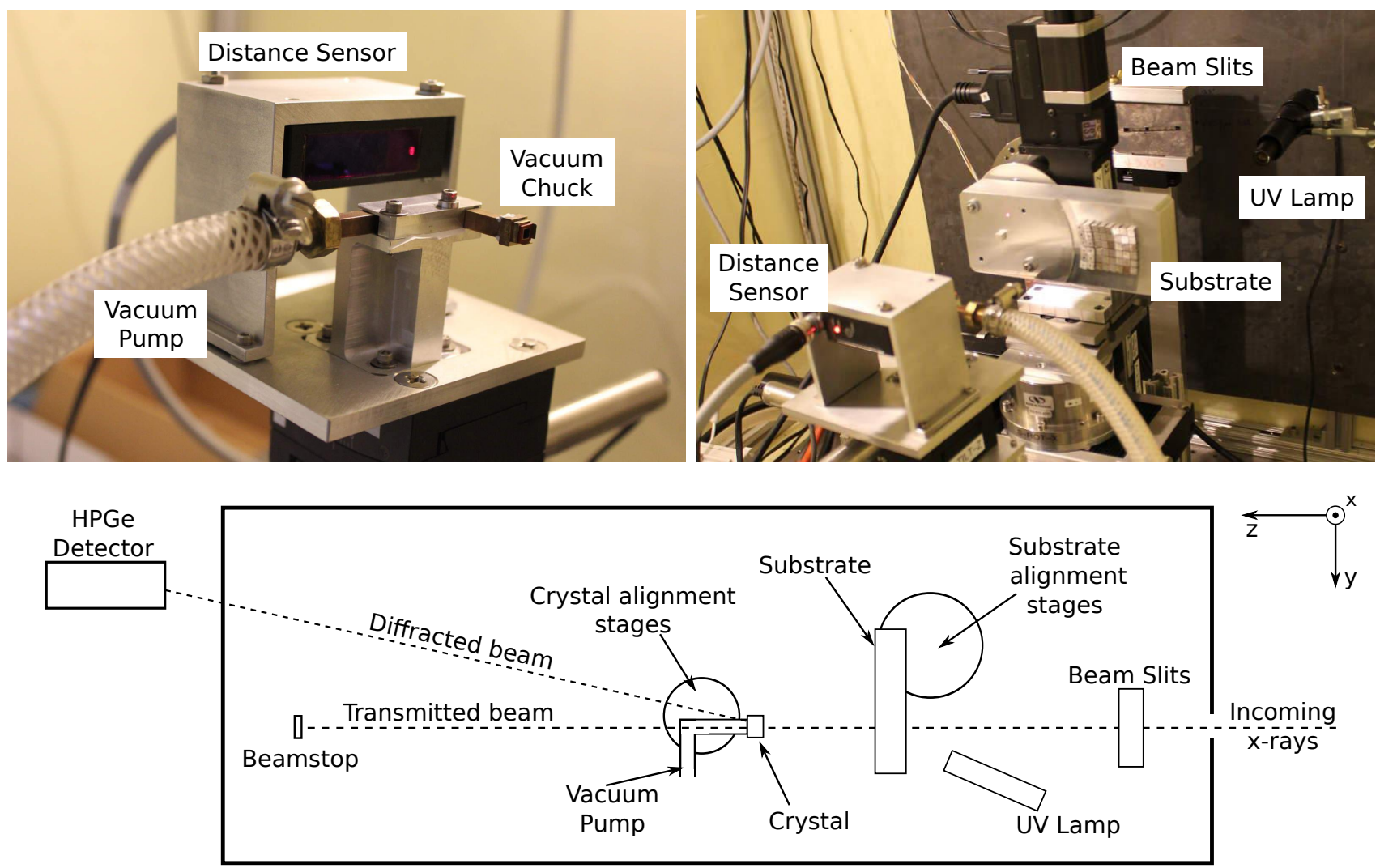

Figure 3. Top left: Vacuum chuck and distance sensor. Top right: Substrate, alignment motors, beam slits and UV lamp. Bottom: Overhead schematic of beamline with definition of axes directions.

Figure 3 shows the dedicated Laue lens assembly beamline at UC Berkeley. The z-axis is defined to be parallel to the x-ray beam, the x-axis is vertically upward and the y-axis is the horizontal axis. An x-ray generator illuminates one side of the assembly station. Beam-slits define the shape and size of the beam to strike the crystal. In this experiment the slits used are $3 \mathrm{~mm}$ high and $4.5 \mathrm{~mm}$ wide.

One set of motors and stages controls the positioning and orientation of the substrate onto which the crystals are glued. Another set of motors controls the fine alignment of the crystal being glued. The motors responsible for it's orientation are aligned so as to always have the crystal in the beam.

\subsection{Choice of Glue}

A glue needs to be stable upon curing so that it does not cause the crystal to become misaligned during the gluing process and also needs to have long term stability to ensure the crystal remains at the intended orientation after being glued. Two glues from Dymax were tested for usability in constructing the lens prototype, one paste (OP-67LS) and one gel (OP-29-GEL). Both glues cure with exposure to ultraviolet light. 


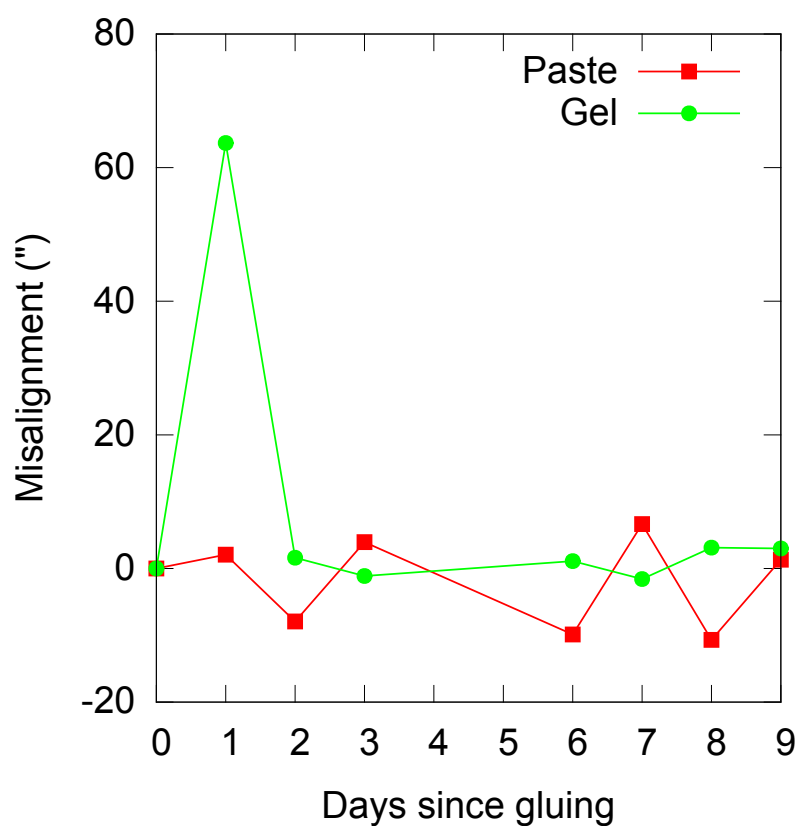

Figure 4. Motion of mirrors over the course of several days with respect to a reference mirror to show the long term stability of different glues.

To test the performance of the gel and the paste, small mirrors were glued onto a larger reference mirror. The substrate was removed and the reference mirror was attached to the rotational stage in its place. The small mirrors were and their relative orientations were tracked through the gluing process using an autocollimator. The gel was found to have larger changes in orientation upon gluing than the paste, either due to the gel's larger shrinkage ( $0.79 \%$ compared to $0.08 \%$ for the paste) or, potentially, the glue not fully curing due to poor illumination. The gel had greater long term stability than the paste as can be seen in Figure 4. The gel was chosen for its long term stability.

\subsection{Gluing Process}

Before gluing can begin, the various components of the experiment must be properly aligned with respect to the x-ray beam:

- The optical table is levelled with respect to the x-ray beam, ensuring it is $38 \mathrm{~cm}$ above the table over the table length.

- The table is aligned with the beam such that the rows of holes are parallel to the beam.

- The substrate alignment crystal is set to $38 \mathrm{~cm}$.

- The substrate rotational axis is aligned with the beam.

- The linear stage of the vacuum chuck is aligned parallel to the beam.

- The tilt stages of the vacuum chuck are aligned to have their centres of rotation inside the beam.

A 3-axis alignment process was developed to ensure the crystal being glued was as accurately oriented with respect to the beam as possible. Both Iron and Aluminium crystals are cubic, this means there are always crystallographic planes perpendicular to those intended for use in the lens. Rotation around the $\mathrm{x}$ axis defines the Bragg angle for the intended crystallographic planes and so rotation around the $\mathrm{z}$ and $\mathrm{y}$ axes defines the Bragg angles for these perpendicular planes. By observing the $n=1$ and $n=-1$ reflections from these perpendicular planes, the central position can be found for the $\mathrm{z}$ and $\mathrm{y}$ rotations. These central positions set the planes parallel to the beam. 

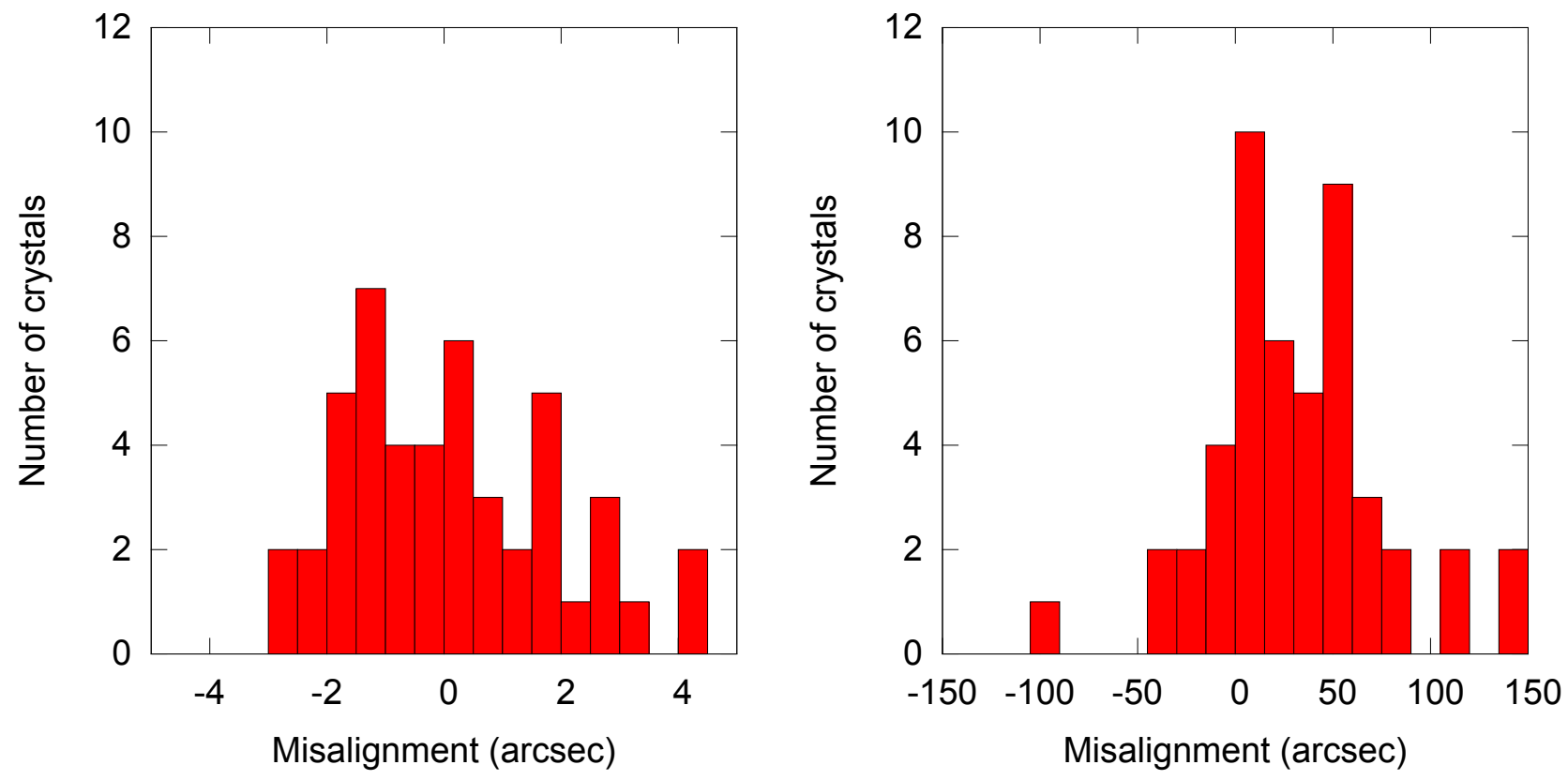

Figure 5. Left: Crystal orientation accuracy achieved before exposing the glue to UV. Right: Orientation accuracy achieved directly after exposing glue to UV.

Once the crystal is aligned about the $\mathrm{z}$ and $\mathrm{y}$ axes, it is oriented about the $\mathrm{x}$ axis. The substrate is oriented so that the site at which the crystal is to be glued is in the beam. Glue is applied to the surface of the crystal and a linear translation stage brings the crystal up against the substrate. A distance sensor is used to bring crystal to $\sim 100 \mu \mathrm{m}$ in front of the substrate. This distance allows the glue to contact both the substrate and the crystal without pushing against each other (which might cause misalignment).

When the crystal is against the substrate fine alignment of the Bragg angle is performed to bring the crystal within $5^{\prime \prime}$ of the desired position. The crystal is then held for $\sim 15$ minutes to see if the glue had become stable. If after 15 minutes, the crystal is still within $5^{\prime \prime}$ of the desired position, then the glue is exposed to 2 minutes of UV light to cure. If the crystal has moved in the allotted time, then it is re-aligned and allowed more time to settle.

Once the curing is complete, the vacuum seal is broken and the vacuum chuck is moved back $0.1 \mathrm{~mm}$ to remove any forces it may contribute to the crystal. A spectrum of the diffraction peak is recorded to measure the motion of the crystal during the curing process.

\section{RESULTS}

Figure 5 shows a histogram of the orientation accuracy achieved for all crystals directly before and directly after curing. The alignment of each crystal before exposure is within $5^{\prime \prime}$ of the desired orientation. After curing, the width of the distribution increases but most crystals remain within one arcminute of the desired orientation.

Table 2 shows the average misalignment for each ring before and after curing the glue along with the standard deviation. Figure 6 shows the effect of the misalignments of the crystals on the spectrum of Ring 0 . There is a noticeable lowering and widening of the diffraction peak. Figure 7 shows a full illumination of the lens by the $\mathrm{x}$-ray beam. The intended peak energy for each ring is marked. The low contribution from the higher rings $(5,6$ and 7 in particular) is due to the sub-optimal collimation. Unfortunately, soon after this spectrum was taken the experiment suffered a critical instrument failure and further data could not be collected. 


\begin{tabular}{ccccc}
\hline & \multicolumn{2}{c}{ Before curing } & \multicolumn{2}{c}{ After curing } \\
Ring & Misalignment(") & $\sigma\left({ }^{\prime \prime}\right)$ & Misalignment(") & $\sigma\left({ }^{\prime \prime}\right)$ \\
\hline 0 & 1.1 & 1.6 & 23.4 & 43.1 \\
1 & -0.2 & 2.2 & 21.1 & 21.3 \\
2 & 0.4 & 1.2 & 90.3 & 48.2 \\
3 & -2.0 & 0.9 & 37.2 & 26.2 \\
4 & -0.1 & 1.5 & 29.6 & 47.8 \\
5 & 0.1 & 1.2 & 19.8 & 37.2 \\
6 & 1.1 & 1.8 & 6.4 & 58.1 \\
7 & 1.2 & 3.4 & 33.9 & 24.7 \\
\hline
\end{tabular}

Table 2. Averages and standard deviations of misalignments in arcseconds for each ring before and after curing the glue.

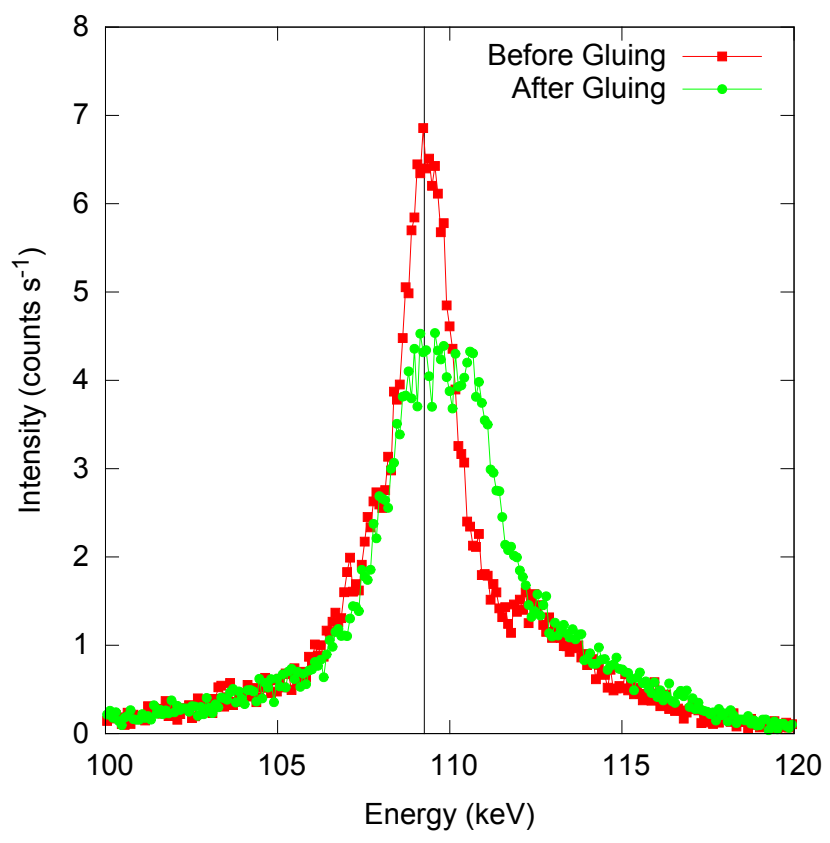

Figure 6. Co-added spectra of all 6 crystals from Ring 0 directly before and directly after curing the glue. The intended central energy is marked with a vertical line $(109.26 \mathrm{keV})$. 




Figure 7. Full illumination of the lens prototype with the intended central peaks for each ring labelled. Label colours represent the crystals composing that ring. Black: Al [200]. Blue: Fe [110]. Red: Fe [200]

\section{CONCLUSIONS}

Compared to the previous iteration of the experiment, ${ }^{15}$ the alignment accuracy has become worse. Previously, an average misalignment of $13.7^{\prime \prime}$ for a single ring had been achieved with a standard deviation of 5.7". The lowest standard deviation achieved in a given ring for the present experiment was $21.3^{\prime \prime}$. This disparity can be largely attributed to the high shrinkage of the glue used. This effect was observed in the glue testing phase (Figure 4).

Although the alignment accuracy of the experiment was worse by a factor of four, the time taken to cure the glue was improved by a factor of 150 . Previously 5 hours were needed to cure the glue before the vacuum chuck could safely be removed. This was lowered to 2 minutes of UV exposure. Approximately 35-40 minutes are required for aligning the crystal before curing the glue. With the new prototype

The Iron and Aluminium crystals provided by Mateck have shown to be promising crystals. As a simple cubic material, Iron offers more crystallographic orientations than base centred cubic or face centred cubic crystals. This makes it very versatile in Laue lens designs. Aluminium, as a low-Z material, can offer good reflectivities at lower energies $(<200 \mathrm{keV})$.

Further study is needed to find a glue which has low shrinkage, long term stability and a short curing time. It has been shown that the UC Berkeley assembly station is capable of highly accurate crystal orientation and very rapid assembly. Combined with an ideal glue, this system could be used to construct a flyable lens.

\section{ACKNOWLEDGMENTS}

LH and CW acknowledge support from Science Foundation Ireland under grant 11-RFP-AST-3188.

\section{REFERENCES}

[1] Iyudin, A. F., Diehl, R., Bloemen, H., Hermsen, W., Lichti, G. G., Morris, D., Ryan, J., Schoenfelder, V., Steinle, H., Varendorff, M., de Vries, C., and Winkler, C., "COMPTEL observations of Ti-44 gamma-ray line emission from CAS A," Astronomy and Astrophysics 284, L1-L4 (Apr. 1994). 
[2] Diehl, R., Siegert, T., Hillebrandt, W., Krause, M., Greiner, J., Maeda, K., Röpke, F. K., Sim, S. A., Wang, W., and Zhang, X., "SN2014J gamma rays from the ${ }^{56} \mathrm{Ni}$ decay chain," Astronomy and Astrophysics 574, A72 (Feb. 2015).

[3] Churazov, E., Sunyaev, R., Isern, J., Bikmaev, I., Bravo, E., Chugai, N., Grebenev, S., Jean, P., Knödlseder, J., Lebrun, F., and Kuulkers, E., "Gamma-rays from Type Ia supernova SN2014J," ArXiv e-prints (Feb. 2015).

[4] Schoenfelder, V., Aarts, H., Bennett, K., de Boer, H., Clear, J., Collmar, W., Connors, A., Deerenberg, A., Diehl, R., von Dordrecht, A., den Herder, J. W., Hermsen, W., Kippen, M., Kuiper, L., Lichti, G., Lockwood, J., Macri, J., McConnell, M., Morris, D., Much, R., Ryan, J., Simpson, G., Snelling, M., Stacy, G., Steinle, H., Strong, A., Swanenburg, B. N., Taylor, B., de Vries, C., and Winkler, C., "Instrument description and performance of the Imaging Gamma-Ray Telescope COMPTEL aboard the Compton Gamma-Ray Observatory," Astrophysical Journal Supplement Series 86, 657-692 (June 1993).

[5] Vedrenne, G., Roques, J.-P., Schönfelder, V., Mandrou, P., Lichti, G. G., von Kienlin, A., Cordier, B., Schanne, S., Knödlseder, J., Skinner, G., Jean, P., Sanchez, F., Caraveo, P., Teegarden, B., von Ballmoos, P., Bouchet, L., Paul, P., Matteson, J., Boggs, S., Wunderer, C., Leleux, P., Weidenspointner, G., Durouchoux, P., Diehl, R., Strong, A., Cassé, M., Clair, M. A., and André, Y., "SPI: The spectrometer aboard INTEGRAL," Astronomy and Astrophysics 411, L63-L70 (Nov. 2003).

[6] Harrison, F. A., Craig, W. W., Christensen, F. E., Hailey, C. J., Zhang, W. W., Boggs, S. E., Stern, D., Cook, W. R., Forster, K., Giommi, P., Grefenstette, B. W., Kim, Y., Kitaguchi, T., Koglin, J. E., Madsen, K. K., Mao, P. H., Miyasaka, H., Mori, K., Perri, M., Pivovaroff, M. J., Puccetti, S., Rana, V. R., Westergaard, N. J., Willis, J., Zoglauer, A., An, H., Bachetti, M., Barrière, N. M., Bellm, E. C., Bhalerao, V., Brejnholt, N. F., Fuerst, F., Liebe, C. C., Markwardt, C. B., Nynka, M., Vogel, J. K., Walton, D. J., Wik, D. R., Alexander, D. M., Cominsky, L. R., Hornschemeier, A. E., Hornstrup, A., Kaspi, V. M., Madejski, G. M., Matt, G., Molendi, S., Smith, D. M., Tomsick, J. A., Ajello, M., Ballantyne, D. R., Baloković, M., Barret, D., Bauer, F. E., Blandford, R. D., Brandt, W. N., Brenneman, L. W., Chiang, J., Chakrabarty, D., Chenevez, J., Comastri, A., Dufour, F., Elvis, M., Fabian, A. C., Farrah, D., Fryer, C. L., Gotthelf, E. V., Grindlay, J. E., Helfand, D. J., Krivonos, R., Meier, D. L., Miller, J. M., Natalucci, L., Ogle, P., Ofek, E. O., Ptak, A., Reynolds, S. P., Rigby, J. R., Tagliaferri, G., Thorsett, S. E., Treister, E., and Urry, C. M., "The Nuclear Spectroscopic Telescope Array (NuSTAR) High-energy X-Ray Mission," The Astrophysical Journal 770, 103 (June 2013).

[7] Lund, N., "A study of focusing telescopes for soft gamma rays," Experimental Astronomy 2, 259-273 (1992).

[8] Frontera, F. and von Ballmoos, P., "Laue Gamma-Ray Lenses for Space Astrophysics: Status and Prospects," X-Ray Optics and Instrumentation, 2010. Special Issue on X-Ray Focusing: Techniques and Applications, id.215375 2010, 14 (2010).

[9] Lindquist, T. and Webber, W., "A focusing X-ray telescope for use in the study of extraterrestrial X-ray sources in the energy range 20-140 keV," Canadian Journal of Physics Supplement 46, 1103 (1968).

[10] von Ballmoos, P., Halloin, H., Evrard, J., Skinner, G., Abrosimov, N., Alvarez, J., Bastie, P., Hamelin, B., Hernanz, M., Jean, P., Knödlseder, J., and Smither, B., "CLAIRE: First light for a gamma-ray lens," Experimental Astronomy 20, 253-267 (Dec. 2005).

[11] Virgilli, E., Frontera, F., Valsan, V., Liccardo, V., Carassiti, V., Squerzanti, S., Statera, M., Parise, M., Chiozzi, S., Evangelisti, F., Caroli, E., Stephen, J., Auricchio, N., Silvestri, S., Basili, A., Cassese, F., Recanatesi, L., Guidi, V., Bellucci, V., Camattari, R., Ferrari, C., Zappettini, A., Buffagni, E., Bonnini, E., Pecora, M., Mottini, S., and Negri, B., "The LAUE project and its main results," ArXiv e-prints (Jan. 2014).

[12] Barriere, N., Tomsick, J., Boggs, S. E., Lowell, A., Wade, C., Jentschel, M., and von Ballmoos, P., "Critical Developments Toward Building Laue Lenses for Gamma-Ray Astronomy," AAS/High Energy Astrophysics Division 13, 123.08 (Apr. 2013).

[13] Zachariasen, W., [Theory of X-Ray Diffraction in Crystals], Dover Publications Inc (1945).

[14] Halloin, H. and Bastie, P., "Laue diffraction lenses for astrophysics: Theoretical concepts," Experimental Astronomy 20, 151-170 (Dec. 2005). 
[15] Barrière, N. M., Tomsick, J. A., Boggs, S. E., Lowell, A., Wade, C., Baugh, M., von Ballmoos, P., Abrosimov, N. V., and Hanlon, L., "Developing a method for soft gamma-ray Laue lens assembly and calibration," Nuclear Instruments and Methods in Physics Research A 741, 47-56 (Mar. 2014).

[16] Barrière, N. M., Tomsick, J. A., Boggs, S. E., Lowell, A., and von Ballmoos, P., "Developing a second generation Laue lens prototype: high-reflectivity crystals and accurate assembly," in [Society of PhotoOptical Instrumentation Engineers (SPIE) Conference Series], Society of Photo-Optical Instrumentation Engineers (SPIE) Conference Series 8147, 1 (Sept. 2011). 